VI.

Aus der chirurgisehen Universitätsklinik in Bern. (Direktor Herr Prof. Dr. Theodor Kocher.)

\title{
Eine experimentelle Arbeit über die Ätiologie der Perityphlitis.
}

Von

Dr. M. Mori, Japan.

(Mit 1 Abbildung im Text und Tafel I.)

Obschon die Erkrankungen in der Gegend des Coecum bereits den alten Ärzten bekannt waren, ist doch die wichtige Erkenntnis der Perityphlitis als eines besonderen Krankheitsbildes eine Errungenschaft des 19. Jahrhunderts. Besonders in seinem letzten Dezennium wurden die Krankheiten des Appendix von verschiedenen Forschern eifrig studiert. Immerhin ist die Ätiologie derselben noch nicht einwandfrei abgeklärt, und man hat immer noch nicht das Interesse verloren, bei Tieren Versuche darüber anzustellen. Hier will ich nicht auf die Literatur der bei Menschen beobachteten Formen der Appendicitis eingehen, sondern begnüge mich mit derjenigen der diesbezüglichen experimentellen Arbeiten.

Roux ${ }^{1)}$ gelang es, bei Schweinen künstliche Appendices durch Plastik vom Coecum zu machen und in dieselben nach Heilung Fremdkörper einzuführen, ohne daß dadurch Veränderungen eintraten; nur einmal sah er Perforation und Kommunikation mit dem Dünndarm.

Roger und Josu (2) erzengten durch Abschnürung des Appendix mit oder ohne Injektion von Colikultur in den Wurmfortsatz ein Krankheitsbild, welches an die gangränöse Appendicitis erinnert. Die beiden Autoren fassen die Appendicitis als eine "Lésion toxi-infectieuse" auf.

Beasusenat ${ }^{3}$ ) studierte in einer Reihe schön angeordneter Experi-

1) Roux, Congrès franç. de chir. Lyon 1894.

2) Roger u. Josué, Revue de méd. 1896. No. 6.

3) Beaussenat, Rev. de gyn. et de chir. abdom. 1897. 2. 
mente die verschiedenen Bedingungen, unter denen sich beim Tier Appendieitis hervorbringen läßt. $\mathrm{Er}$ teilt seine Versucle in zehn Gruppen ein und erzeugt in seiner fünften Gruppe die Infektion durch intravenöse Injektion von Colikultur nur bei gleichzeitigem Trauma des Wurmfortsatzes eine Ulceration in diesem. Er zieht aus seinen Versuchen folgende Schlüsse: Die Perityphlitis ist immer oder fast immer die Folge einer Infektion, die zwar ausnahmswesse auf dem Wege der Blut- und Lymphbahn erfolgen kann, gewöhnlich aber vom Darmlumen aus stattfindet. Meist ist Bacterium coli die Ursache der Erkrankung. Damit die Bakterien pathogen werden können, muß die normale Lebensfäligkeit der Schleimhaut gestört oder die den Schutz bewirkende phagocytäre Tätigkeit derselben aufgehoben sein.

de Klecki ${ }^{1}$ ) betont die Infektion als ätiologisches Moment noch melır.

Mühsam 2) machte eine Reihe von Versuchen an Kaninchen, wobei er hauptsächlich Zirkulationsstörungen hervorrief und faßt die Ergebnisse derselben in den Satz zusammen: bei Kaninchen sei es ihm gelungen, nur Appendicitis gangraenosa zu erzeugen. In den Versuchen, bei denen die Herbeiführung der Wurmfortsatzgangrän gelang, handelte es sich immer um totale oder partielle Unterbindung der zuführenden Gefäße, wodurch der Processus bald ganz, bald nur teilweise nekrotisch wurde. Es hat sich vor allem aber erwiesen, daß bei Kaninchen durch die Gangrän des Wurmfortsatzes keine entzündlichen Prozesse in den benachbarten Teilen desselben verursacht werden, daß die Prozesse vorwiegend lokaler Natur waren.

S. M. Lipawski ${ }^{3}$ ) machte folgende experimentelle Arbeit, - 22 Versuche mit Einführung von Streptococcus- und Bact. Coli-Kulturen in die Art. appendicularis und in die Wurmfortsatzhöhle. Dabei hat er immer die Unterbindung oder Abquetschung der Wand, oder chemische Reizung derselben vorgenommen. Es hat sich in einigen Versuchen gezeigt, daß die Injektion von abgeschwächter Kultur des Streptococeus in die Arteria keine sichtbaren Veränderungen hervorrief.

A. L. Polenow ${ }^{4}$ ) machte 15 Experimente an Kaninchen, von denen zehn genau beschrieben und schematisch abgebildet sind. Der Wurmfortsatz wurde total unterbunden oder verengt, geknickt, $180^{\circ} \mathrm{um}$ seine Achse gedreht, endlich wurden die Blutgefäße des Wurmfortsatzes unterbunden. Er will experimentell die verschiedensten am Menschen beschriebenen Appendicitisformen beobachtet haben.

J. Nikolaysen ${ }^{5}$ ) rief in seiner ersten Versuchsreihe eine lokale Disposition im Wurmfortsatz durch subkutane Einspritzung von Toxin des Bacterium coli hervor, nachdem er sich davon überzeugt hatte, daß nach einer solchen Einspritzung Schwellung der Schleimhaut in dem Appendix erfolgte. Zwei der Tiere boten die für die Streptococcuswirkung charakteriștische Veränderung dar. In seiner zweiten Versuchsreihe wurden die

1) de Klecki, Contribution à la pathogénie de l'appendicite. Annales de l'Institut Pasteur: 1899. p. 480.

2) Mühsam, Deutsche Zeitschr. f. Chirurgie. 55. Bd. 1. u. 2. Heft. 1900.

3) S. M. Lipawski, Dissert. St. Petersburg 1901.

4) A. L. Polen ow, Russki, chir. Archiv. 1902. Nr. 3.

5) J. Nikolaysen, Nord. med. Artik. Bd. XXXIV. III. F. 
'Tiere zunächst mit Strepptococcuskultur und vier Tage später mit $20 \mathrm{ccm}$ einer zwei Tage alten Bouillonkultur von Bacterium coli gefüttert. Hier zeigten die pathologischen Veränderungen einen anderen Charakter.

Meine Experimente wurden nur bei Kaninchen gemacht, trotzdem wir wissen, daß die Blutversorgung des Appendix beim Kaninchen im Vergleich zu derjenigen des Menschen eine sehr gute ist und sich infolgedessen Kaninchen nicht gerade zu Experimenten eignen. Ich habe aber gefunden, daß spontane Appendicitis bei Kaninchen vorkommt, worüber kürzlich eime Mitteilung in "Grenzgebieten zwischen Medizin und Chirurgie" erschienen ist.

Schon längst ist man der Ansicht, daß sich eine Appendicitis bei Menschen an Eiterungen und Entzündungen anderer Körperteile anschließen kann; so sieht man bei Individuen, die an klinisch schwer verlaufender Streptokokken-Angina gelitten haben, Entzündungen des Wurmfortsatzes auftreten. Als bekanntes Beispiel wird Gambettas Krankheit genannt; er fiel einer Appendicitis zum Opfer, welche während der Heilung einer fistulösen Wunde an der Hand und am Vorderarm aufgetreten war. Im allgemeinen aber sind hämatogene Infectionen des Appendix nach den meisten Autoren selten.

Ich hatte nun in erster Linie die Absicht, das Vorkommen dieser hämatogenen Form experimentell nachzuweisen, und zwar ohne an Ort und Stelle irgend welche Schädigung des Wurmfortsatzes herbeizuführen. Zu dem Zweck wurde zunächst die Arteria appendicularis benutzt, um die infektiöse Masse in die Blutbahn zu bringen.

Bevor ich auf die Schilderung meiner Experimente komme, möchte ich mir erlauben, einige Worte über die Technik der Operation vorauszuschicken. Die Arteria appendicularis des Kaninchens ist immer sehr dünn, trotzdem der Appendix bei ihm sehr ausgesprochen ist. Sie blutet schon beim Einstechen der Spritznadel und noch viel mehr bei Herausnahme derselben. Um die Nachblutung stillen zu können, lege ich nach der Prozedur eine Peritonealnaht (Überzugsnaht) an, damit die Arterienwandung leicht angedrückt und eine weitere Blutung gehindert wird. Da aber diese Naht das Gefäßlumen nicht komprimiert, so geht die Blutzirkulation trotzdem ganz gut von statten. Die Spritznadel wird kurz vor dem Gebrauch mit physiologischer Kochsalzlösung gefüllt, um den Rücklauf des Blutes während des Stechens in die Arteria zu hindern, da sonst das rückströmende Blut ziemlich schnell gerinnen und dadurch das Einspritzen der bakterienhaltigen Flüssigkeit unmöglich machen würde.

Die von mir gebrauchte krumme Nadel war von feinster Nummer. Ich will hier ausdrücklich bemerken, daß die Injektion in die Arteria 
appendicularis ungemein schwierig ist. Bei einem kleinen Kaninchen ist die Operation überhaupt unmöglich; selbst bei größeren gelingt sie nur sehr selten; ich möchte fast sagen, eine glatte Operation $d .1$. ein richtiges Eindringen in die Arteria ist so zu sagen als Zufall zu betrachten.

Ich habe bei ganz gelungenen Injektionen mehrmals beobachtet,

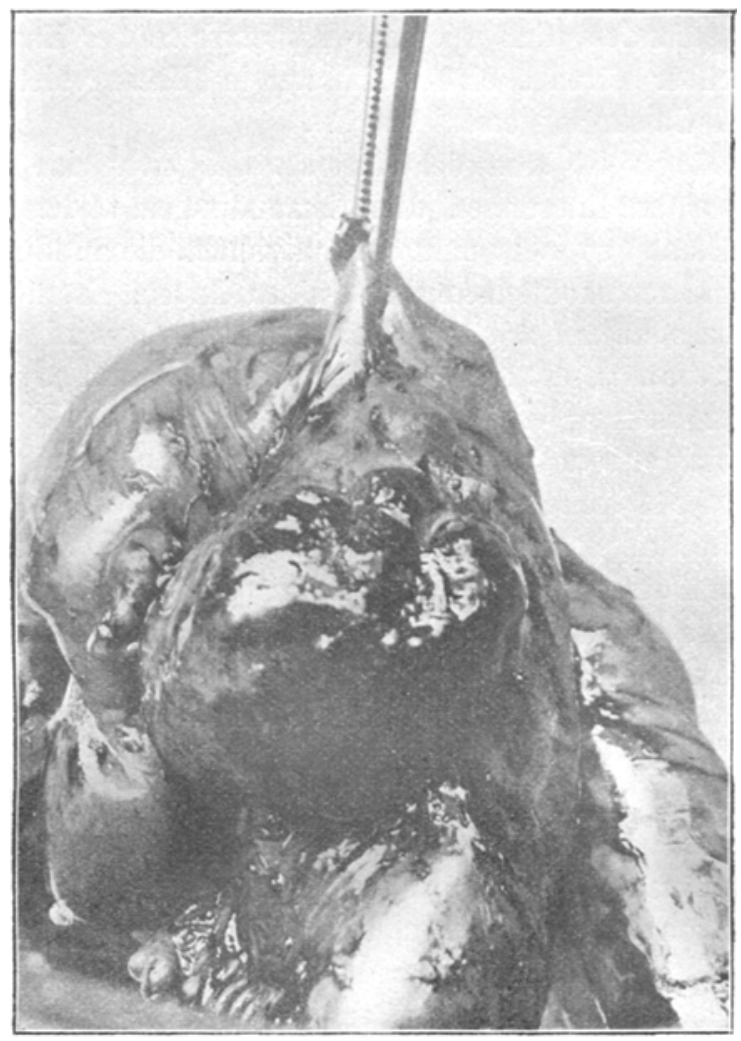

Tuberkulöse Entzündung des Sac. rot. von der Schleimhautseite gesehen.

daß während der Injektion das Injektionsmaterial von dem Blutstrom fortgeschwemmt wurde. Dabei fand auf eine relativ weite Distanz eine Vermischung von Blut und Injektionsmaterial nicht statt, so daß der Inhalt der Arteria durch die dünne Wand hindurch auf kurze Strecken abwechslungsweise rot und farblos erschien. Dieses Verhalten des Arterieninhaltes möchte ich als sicheres Kriterium dafür bezeichnen, daß die Injelstion wirklich gelungen ist. Selir oft bildete sich ein Hämatom an der Stelle der Einspritzung, wodurch das Re- 
sultat illusorisch gemacht wurde. Am vorteilhaftesten wäre es, wenn man die Arteria appendicularis direkt nach ihrer Abzweigung von der Arteria ileocolica benutzen könnte. Um jedoch zu diesem Stamme gelangen zu können, muß man abpräparieren, wodurch leider eine nicht zu stillende Blutung der Arteria wie auch der Venen entsteht - eine Folge der leichten Zerreißbarkeit der Gefäßwandungen des Kaninchens - und von einer allfälligen beabsichtigten Operation wird keine Rede mehr sein können.

Die von mir verwendeten Materialien sind 1. Colibacterium 1), 2. Streptokokkus, 3. Staphylococcus aureus, 4. Proteus, welchen ich bei einer spontanen Appendicitis eines Kaninchens gefunden habe, (diese vier in Bouillonkultur) 5. frischer verdünnter Eiter, dessen Herkommen und Bakteriengehalt genau bei jedem Versuch angegeben werden wird.

Die gesamte Zahl der Versuche belief sich auf 66 . Ich werde aber hier nur diejenigen in Betracht ziehen, bei denen die Injektion gelungen ist, und die unsicheren und mißlungenen bei Seite lassen. Meine Operationen wurden unter den strengsten aseptischen Kautelen ausgeführt. Zunächst gehe ich auf die Beschreibung der ersten Versuchsreihe über.

Versuch 5. 1.6. Mai 1903. Einspritzung von Staphylococcus aureus $0,3 \mathrm{ccm}$ in die Arteria appendicularis.

28. Mai wurde das Kaninchen getötet. Sektionsbefund: In der Peritonealhöhle findet sich keine Veränderung; unter der Serosa des Appendix ein weißer Herd; er ist reißkorngroß; der Eiter sehr zäl. Unweit der Appendixspitze sieht man zwei hämorrhagische Stellen; eine von $0,7 \mathrm{~cm}$, die andere von nur 0,5 cm Durchmesser. An der Schleimhaut finden sich denjenigen der Serosaseite entsprechende, aber viel ausgedehntere Hämorrhagien. Die eine ist nach innen aufgebrochen und bildet einen geschwürähnlichen Schlitz.

Die bakteriologische Untersuchung ergibt folgendes: Aus dem obenerwähnten Eiterherd ließen sich Colibazillen und lange Bazillen, welche saprophytischer Natur sein dürften, züchten. Diese zutage getretenen Erscheinungen und der Umstand, daß eine listologische Untersuchung nicht möglich war, läßt uns im Zweifel darüber, ob hier das Experiment gelungen ist, oder ob wir es nur mit einer nicht beabsichtigten operativen Infektion zu tun hatten.

Versuch 11. 20. Oktober 1903. Einspritzung einiger Tropfen von Proteus in die Art. appendic.

6. November Kaninchen getötet. Sektionsbefund: Auf dem Appendix finden sich zwei kleine Abszesse, außerdem mehrere ziemlich zerstreut liegende hämorrhagische Stellen; auf den entsprechenden Stellen der Schleim-

1) Cf. M. Mori, Spontane Appendicitis bei einigen Tierspezies. 1903. 12. Bd. Heft 5. S. 640. Mitteilungen aus den Grenzgebieten zwischen der Medizin $u$. Chirurgie. S. 640. Vergl. auch in diesem Hefte Fig. 3. 
hautseite zeigen sich ebenfalls Hämorhagien, von denen jedoch nur etwa ein Drittel geschwürig zerfallen sind.

Aus den Appendixherden wie auch aus dem Appendixinhalt lassen sich nur Colibazillen züchten.

Eine histologische Untersuchung konnte aus dem gleichen Grunde wie oben nicht vorgenommen werden.

Versuch 16. 5. Januar 1904. Einspritzung einiger 'Tropfen Eiter' aus einem Abszeb am Oberschenkel eines Menschen in die Art. appendic.

Bei dieser Injektion konnte ich das obenerwähnte Kriterium für das Gelingen der Injektion sehr deutlich wahrnehmen.

Am 18. Januar Relaparotomiert. Keine Veränderungen.

Versuch 18. 17. Januar 1904. Einspritzung von $0,3 \mathrm{ccm}$ Eiter aus einer lokalen Phlegmone (Streptomykose) am Oberarm eines Mannes in Art. appendic. Auch bei dieser Injektion war die angeführte mangelhafte Vermischung des Arterieninhaltes zu konstatieren; keine Hämatombildung.

18. Januar ging das Tier ein. Sektionsbefund: Im ganzen Bereich des Appendix findet sich eine hochgradige Entzündung. Dieselbe zeichnet sich dadurch aus, daß der Appendix hämorrhagisch infiltriert, stark geschwollen und zum Teil schon gangränös geworden ist. An der Oberfläche des Appendix zeigen sich stellenweise Fibrinauflagerungen (siehe Fig. 1 auf Tafel I). Der dem Appendix nahe liegende Dünndarm, dessen Gefäße zum Teil anch mit denjenigen des Appendix gemeinschaftlich versorgt werden, ist ziemlich stark an der Entzündung beteiligt. Am Coecum, nahe beim Appendix, finden sich zwei große Hämorrhagien. Die Schleimhaut des Appendix ist im ganzen sehr gerötet. An einigen Stellen derselben sieht man weiflich-gelbe, nekrotisch aussehende Partien, die ziemlich umschrieben und von Hämorrhagien umgeben sind.

Die bakteriologische Untersuchung ergibt: Aus den entzündeten Herden und aus dem Appendixinhalt lassen sich Colibazillen und Streptokokken und aus dem Herzblut Streptokokken züchten.

Die histologische Untersuchung zeigt: Fehlende Kernfärbbarkeit usw.

Bei den Versuchen Nr. 5 und Nr. 14 kann ich nicht mit Sicherheit behaupten, ob die Injektion wirklich gut vor sich gegangen ist. Außerdem konnte ich keine histologischen Untersuchungen der bereits angegebenen Gründe wegen vornehmen. Es ist fraglich, ob es sich dort wirklich um eine artifizielle Appendicitis oder einen Zufall gehandelt hat. Dagegen liegt bei dem Versuch 18 kein Zweifel vor, ja ich kann sicher behaupten, daß hier eine artifizielle und zwar gangränöse Entzündung vorliegt.

Wie ich oben hervorhob, ist es bei dieser Methode sebr schwierig, das Arterienlumen richtig zu treffen; deshalb habe ich bei späteren Versuchen meine Methode modifiziert, indem ich die Arteria mesenterica superior benutzte und zwar aus zwei Grïnden: Einerseits ist man dabei sicher, die Injektion richtig ausführen zu können, und andererseits läßt sich dabei beobachten, ob der Appendix mehr zu Entzïndungen disponiert ist, als die anderen Darmabschnitte. Meine 
Technik bei dieser Operation war folgende: Man macht zunächst einen Medianschnitt am Abdomen, der ganze Darm samt Mesenterium wird herausgenommen und nach unten geklappt, damit die Arteria mesenterica deutlich zu Tage trete. Bei diesem Verfahren braucht man keine Peritonealnaht anzulegen, da die Arteria genügend dick ist und eine Nachblutung selten erfolgt. Hier wie dort kam irgend welche Schädigung des Appendix nicht vor.

Versuch 20. 31. Oktober 1903. Einspritzung von 1,0 ecm Colibazillenkultur in Arteria mesenterica superior.

2. November. Das Tier ging ein. Sektionsbefund: An dem Appendix finden sich mehrere punktförmige Blutungen. Der Appendix ist sehr atrophisch. Am Coecum findet man stellenweise ganz zerstreut auch Blutungen unter der Serosa. Von einer allgemeinen Peritonitis ist keine Rede.

Versuch 21. 5. November 1903. Einspritzung von 0,5 cem Streptokokken in Art. mesent. sup.

8. November. Kaninchen eingegangen. Sektionsbefund: Der Appendix hochgradig hämorrhagisch, der Sacculus rotundus ${ }^{1)}$ ebenso. Der ganze Dünndarm nebst Sace. rot. ist mit fibrinösen Massen bedeckt, seine Schlingen sind miteinander verklebt. An der Oberfläche der Leber findet sich auch Fibrinauflagerung. Zweifellos handelt es sich hier um eine Peritonitis. Meiner Ansicht nach ist die Entzündung der Darmwandung primär und die Peritonitis sekundär.

Versuch 22. 6. November 1903. Einspritzung von 0,5 ccm Staphylococcus aureus in Art. .mesent. sup.

9. November. Kaninchen eingegangen. Sektionsbefund: In der Peritonealhöhle findet sich keine nennenswerte Veränderung. Der Appendix ist ganz unbedeutend injiziert.

Versuch 23. 12. November 1903. Einspritzung einiger Tropfen Proteus in Art. mesent. sup.

21. November. Relaparotomiert. Keine Veränderungen.

Versuch 24. 13. November 1904. Einspritzung einiger Tropfen von Proteus in Art. mesent. sup.

23. November. Tier getötet. Sektionsbefund: Der Appendix vollständig übersät von zahlreichen subserösen Hämorrhagien von verschiedener Größe. Außerdem zeigen sich am Appendix mehrere kleine, etwa mohnkorngroße Erhabenheiten, die dem Aussehen nach Miliarabszesse darstellen können.

Die histologische Untersuchung des Schnittpräparates ergibt folgendes Resultat: Die miliarabszeßähnlich aussehenden Gebilde enthalten keine Eiterkörperehen, sondern bestehen hauptsächlich aus Epitheloidzellen und Fremdkörperriesenzellen, zwischen denen ungefärbte, glänzende Massen lagen. Welcher Art diese Fremdkörper waren, ließ sich nicht mit Sicherheit feststellen; jedenfalls rühren sie von Unreinigkeiten beim Einspritzen her. In

1) Am Anfang des Coecum öffnet sich mit weiter Mündung ein woißlicher, ovaler, $2 \mathrm{~cm}$ langer drüsiger Anhang: Der Sacculus rotundus, dicht neben dem Übergange des Dünndarms in den Dickdarm. Dic Wandung desselben enthält dichtgedrängte Lymphfollikel. 
den Follikeln finde ich überall Blutungen, nirgends aber gelappt kernige Lenkozyten.

Die bakteriologische Untersuchung ergibt folgendes: Aus den oben erwähnten erhabenen Stellen und aus dem Appendixinhalt läßt sich Staphylococcus albus züclten; aus dem letzteren überdies Colibacillus, die Hämorrhagien weisen keine Bakterien auf.

Versuch 25. 14. November 1903. Einspritzung einiger Tropfen von Staphylococcus aureus in Art. mesent. sup.

1. Dezember. Relaparotomiert. Nirgends Veränderungen.

Versuch 26. 14. November 1903. Einspritzung einiger Tropfer Koli in Art. mesent. sup.

25. November. Tier eingegangen. Sektionsbefund: keine Veränderungen.

Versuch 27. 17. November. Einspritzung einiger Tropfen Streptokokken in Art. mesent. sup.

8. Dezember. Kaninchen getötet. Keine Veränderungen.

Versuch 29. 23. November 1903. Einspritzung von 0,3 cem Eiter einer operierten Warzenfortsatzentzündung in Art. mesent. sup. Der Eiter enthält Colibazillen.

12. Dezember Relaparotomiert. Nirgends Veränderungen.

Versuch 30. 24. November. Einspritzung von 0,3 cem Eiter des obigen Falles in Art. mesent. sup.

25. November. Tier verendet. Sektionsbefund: Das Coecum wie auch der Dickdarm bis zum Rectum zeigen frische, feine Hämorrhagien, während Appendix, Sacculus rotundus und Dünndarm ganz verschont geblieben sind. Keine Peritonitis.

Versuch 31. 26. November 1903. Einspritzung von $0,3 \mathrm{ccm}$ Proteus in Art. mesent. sup.

6. Dezember. Tier eingegangen. Sektionsbefund: Am Coecum verschiedene Hämorrhagien, zum Teil vereitert, eine sogar gangränös geworden. Appendix und Sac. rot. zeigen auch hämorrhagische Erscheinungen, aber nicht in dem Maße, wie das Coecum.

Bakteriologische Untersuchung: Aus gangränösen Partien lassen sich Colibazillen, aus dem Coecuminhalt Coli und Subtilis züchten; im Herzblut läßt sich kein Bacterium konstatieren.

Versuch 32. 26. November 1903. Einspritzung von 0,5 ccm Proteus in Art. mesent. sup.

14. Dezember. Tier getötet. Sektionsbefund: Appendix und Sac. rot. zeigen keine Veränderungen, der Dünndarm dagegen zeigt verschiedene Blutungen.

Versuch 33. 27. November 1903. Einspritzung von 0,5 ccm Eiter aus dem Eiterherd einer Nierentuberkulose eines Menschen in Art. mesent. sup. Der Eiter enthält nur Tuberkelbazillen.

15. Januar 1904. Relaparotomiert. Weder in der Bauchhöhle noch in den Lungen Anzeichen von Tuberkulose.

Versuch 34. 28. November 1903. Einspritzung von 0,4 cem Eiter von einem an Appendicitis leidenden Menschen, welcher denselben einige Tage nach einer Radikaloperation durch das Rectum entleerte, in Art. mesent. sup. Der Eiter enthält liauptsächlich Colibazillen.

17. Dezember. Kaninchen relaparotomiert. An einer Stelle des Coecum 
finde ich eine leichte Blutung, die aber durch das Reponieren des Coecum bei der Operation entstanden zu sein scheint.

Versuch 35. 29. Dezember 1903. Einspritzung vou 0,4 ccm Eiter des obigen Falles in Art. mesent. sup.

21. Dezember. Tier getötet. Keine Veränderungen.

Versuch 37. 4. Dezember 1903. Einspritzung von 0,5 ccm Eiter aus der Kniegelenksentzündung eines Mannes (Staphylococcen albi enthaltend).

6. Dezember. Tier relaparotomiert. Keine Veränderungen.

Versuch 38. 6. Dezember 1903. Einspritzung von 0,5 ccm Eiter aus einer Strumitis in Art. mesent. sup. Derselbe war steril.

23. Dezember. Tier relaparotomiert. Keine Veränderungen.

Versuch 39. 9. Dezember 1903. Einspritzung von 0,5 ccm Proteus in Art. mesent. sup.

15. Januar 1904. Relaparotomiert. Keine Veränderungen.

Versuch 40. 21. Dezember 1903. Einspritzung von 1,0 ccm Proteus in Art. mesent. sup.

22. Dezember. Tod des Tieres. Sektionsbefund: In der Bauchhöhle eine Menge Blut. Am Appendix finden sich zahlreiche Hämorrhagien, ebenso am Sac. rot.

Vers u ch 41. 22. Dezember 1903. Einspritzung von 0,5 ccm Eiter aus einem Abszeß am Oberschenkel eines Mannes (Staphylomykose) in Art. mesent. sup.

29. Dezember. Tod des Tieres. Sektionsbefund: Ein Teil des Coecum ist gerötet und mit fibrinösen Massen bedeckt; es handelt sich hier um eine lokale Peritonitis.

Versuch 42. 22. Dezember 1903. Einspritzung von 0,5 ccm Eiter von dem gleichen Fall, wie oben, in Art. mesent. sup.

26. Dezember. 'Tier eingegangen. Sektionsbefund: An der Übergangsstelle des Coecum in den Dickdarm etwa $1 \mathrm{~cm}$ entfernt vom Sac. rot. finden sich zwei ovale hämorrhagisch infiltrierte gangränöse Partien von ungefähr $0,5 \mathrm{~cm}$ Durchmesser, die zirka $1,5 \mathrm{~cm}$ von einander abstehen. Eine derselben zeigt in der Mitte eine weißlichgelbe nekrotisch aussehende Stelle, deren Grölje etwa ein Drittel der Hämorrhagie erreicht. Die andere zeigt auch ähnliche Verhältnisse, aber in geringerem Maße. Die Schleimhaut des Coecum zeigt diesen Stellen entsprechend Geschwürsbildung. Dem Geschwürsgrund ein fest haftendes Blutcoagulum aufsitzend. Die nekrotischen Stellen sind von Hämorrhagien umgeben (cf. Fig. 2 auf Tafel I). Im übrigen zeigen sich einige Hämorrhagien auch am Coecum. Appendix und Sac. rot. sind dabei gar nicht beteiligt.

Bakteriologische Untersuchung: Aus dem Herd wie auch aus dem Coecuminhalt lassen sich Colibazillen und Diplo-Streptokokken, aus letzterem Staphylokokken und Subtilis züchten. Die eben erwähnten Diplokokken zeigen keine Ähnlichkeit mit den gespritzten Streptokokken; im Herzblut lassen sich keine Bakterien nachweisen.

Versuch 46. 26. Dezember 1903. Einspritzung von 0,6 ccm Proteus in Art. mesent. sup.

16. Jauuar 1904. Relaparotomiert. Keine Veränderungen.

Versuch 47. 28. Dezember 1903. Einspritzung von 0,5 ccm Eiter von einer Lymphangitis (Streptokokken enthaltend) in Art. mesent. sup. 
18. Januar 1904. Relaparotomiert. Keine Veränderungen.

Versu ch 49. 30. Dezember 1903. Einspritzung von $0,5 \mathrm{ccm}$ Eiter aus dem Abszeß am Oberschenkel eines Menschen (Staphylococcus aureus enthaltend) in Art. mesent. sup.

5. Januar 1904. Tod des 'Tieres. Sektionsbefund: Am Appendix finden sich mehrere Hämorrhagien; keine Eiterung. Der Sac. rot. zeigt keine Veränderungen. Das Coecum etwas hyperämisch.

Vers u ch 50. 31. Dezember 1903. Einspritzung von 0,5 ccm Eiter des eben erwälinten Falles in Arrt. mesent. sup.

16. Januar 1904. Relaparotomiert. Keine Veränderungen.

Versuch 52. 2. Januar 1904. Einspritzung von 0,5 ccm Eiter, wie oben, in Art. mesent. sup.

6. Januar 1904. Kaninchen eingegangen. Sektionsbefund: An dem Dickdarm zeigen sich große Veränderungen. - Ein Teil des Dickdarms einige Zentimeter vom Coecum abstehend, ist stark entzündet $d$. h. ein ganzer Abschnitt des betreffenden Darms ist auf eine Länge von zirka $2 \mathrm{~cm}$ zirkulär nekrotisiert.

Eine zweite entzündete Stelle findet sich noch weiter unten etwa $3 \mathrm{~cm}$ von der ersten entfernt; dieser Abschnitt ist mit dem Mesenterium des Coecum an den Dickdarm herangezogen. Die Schleimhaut der beiden entzündeten Teile zeigt Geschwürsbildung. Keine peritonitisehen Erscheinungen.

Die histologische Untersuchung ergibt folgendes: Ausgedehnte nekrotisierende Partien, fehlende Kernfärbung, Blutungen usw.

Bakteriologische Untersuchung: Aus dem nekrotischen Herd lassen sich Coibacillus und Staphylococcus albus und aus dem Coecuminhalt Colibacillus züchten. Im Herzblut lassen sich keine Bakterien konstatieren.

Versuch 53. 2. Januar 1904. Einspritzung von $0,5 \mathrm{cem}$ Eiter des gleichen Falles in Art. mesent. sup.

18. Januar 1904. Kaninchen getötet. Keine Veränderungen.

Versuch 54. 3. Januar 1904. Einspritzung von 0,5 ecm Eiter des gleichen Falles in Art. mesent. sup.

4. Januar. Tier eingegangen (etwa nach 20 Stunden). Sektionsbefund: Der Appendix ist gangränös geworden; die Schleimhaut desselben zeigt mehrere Geschwüre. An einem Teil des Coecum und an verschiedenen Stellen des Dünndarms finden sich in Gangrän übergehende Partien. In der Bauchhöhle läßt sich in geringerer Menge blutige Flüssigkeit konstatieren. Es handelt sich offenbar hier um eine beginnende Peritonitis, die aber Folge der Entzündung der Darmwand sein kann.

Die histologische Untersuchung ergibt folgendes: An den verschiedenen Stellen der Appendixwandung finden sich Blutungen und fehlende Kernfärbung usw.

Bakteriologische Untersuchung: Aus dem Appendixherd lassen sich Staphylococcus aureus und Bacterium coli, ans dem Appendixinhalt Bacterium coli züchten. Im Herzblut läßt sich Staphylococcus aureus konstatieren.

Versuch 55. 5. Januar 1904. Einspritzung von 0,3 ccm Eiter aus gleichem Fall, wie oben in Art. mesent. sup.

8. Januar. Tod des Tieres. Sektionsbefund: Es sind keine wesentlichen Veränderungen nachzuweisen. Die Todesursache muß der allgemeinen Sepsis zugeschrieben werden. 
Versuch 58. 7. Januar 1904. Einspritzung von $0,3 \mathrm{ccm}$ Eiter aus einer Streptomykose eines Menschen in Art. mesent. sup.

19. Januar. Relaparotomiert. Keine Veränderungen.

Versueh 59. 7. Januar 1904. Einspritzung von 0,3 ecm Eiter aus obigem Falle in Art. mesent. sup.

11. Januar. Tod. Sektionsbefund: Eine rundliche Hämorrhagie auf dem Appendix von zirka $3 \mathrm{~mm}$ Durchmesser. Die Schleimhant weist keine Veränderungen auf. An den peripherischen Teilen des Sac. rot. findet sich eine große unregelmäßig gestaltete Hämorrhagie. Am Coecum haften fibrinöse Massen. An einer Stelle des Dünndarms findet sich ein ausgedehnter intensiv geröteter zum Teil hämorrhagiseh gewordener Abschnitt, auf dem Fibrinmassen lagern. Im Mesenterium des Dünndarms findet sich eine recht umfangreiche Hämorrhagie.

Bakteriologische Untersuchung. Aus der hämorrhagischen Stelle und aus dem Appendixinhalt lassen sich nur Colibazillen züchten.

Versuch 60. 8. Januar 1904. Einspritzung von $0,3 \mathrm{ccm}$ des gleichen Eiters in Art. mesent. sup.

22. Januar 1904. Tier getötet. Keine Veränderungen.

Versuch 62. 15. Januar 1904. Einspritzung von 0,8 cem Streptokokkenkultur, die auch bei Versuch 56 verwendet wurden.

16. Januar 1904. Tod. Sektionsbefund: Am Coecum findet man zwei voneinander abstehende ausgedehnte Hämorrhagien. Der Appendix weist keine Veränderungen auf.

Versuch 63. 15. Januar 1904. Einspritzung von 0,7 cem Staphylokokkenkultur.

23. Januar 1904. Tier getötet. Keine Veränderungen.

Versuch 66. 16. Januar 1904. Einspritzung von 0,5 ccm Eiter vom Abszeb eines Mannes, Streptokokken enthaltend.

17. Januar 1904. Tod des Tieres. Keine Veränderungen. Die Todesursache muß allgemeiner Sepsis zugeschrieben werden.

Die Versuchstiere der zweiten Kategorien zeigen uns folgende Resultate:

Hämorrhagie am Appendix . . . . . . . . . . . . . 2

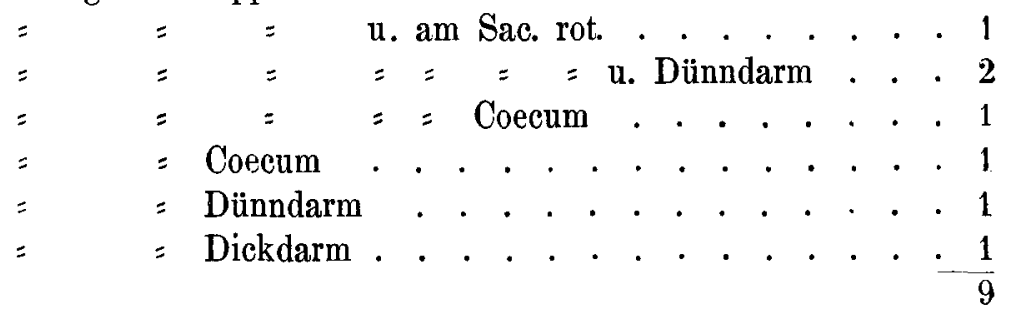

Gangrän am Appendix, Coecum und Dünndarm . . . . . . 1

$=\quad=$ Coecum . . . . . . . . . . . . . 2

$=\quad=$ Dickdarm . . . . . . . . . . . . . . $\frac{.1}{4}$ 
Auf das Vorhandensein von Blutungen in den verschiedenen Darmabschnitten möchte ich kein großes Gewicht legen. Da ich nämlich mein Hauptaugenmerk auf den Appendix richtete, so ist es wohl möglich, daß jeh Blutungen an anderen Darmteilen übersehen habe. Ich bin infolgedessen nicht in der Lage, mit Bestimmtheit zu behaupten, daß die Blutungen in der Wand des Appendix häufiger vorgekommen sind, als in den übrigen Darmabschnitten.

Von den vier Fällen, welche Gangrän zeigten, wurden drei mit frischem Eiter injiziert, einmal Eiter mit Streptokokken, zweimal Eiter mit Staphylococcus albus. Man kann hier nicht ohne weiteres nachweisen, welcher Eiter der wirksamste gewesen ist, um eine Gangrän hervorzurufen, da die Verdünnung jedesmal verschieden war. Jedenfalls aber war dazu eine ziemlieh starke Dosis erforderlich. Ferner geht aus gelungenen Versuchen, deren Zahl allerdings keine große ist, hervor, daß der frische Eiter eher imstande zu sein scheint, Gangrän hervorzurufen, als eine Reinkultur von verschiedenen Eitererregern.

Trotzdem bei einer Anzahl von Versuchstieren eine gleich große und zwar bedeutende Menge virulenter Materie injiziert wurde, so konnte doch bei der Mehrzahl der Fälle auch nicht die geringste schädliche Wirkung konstatiert werden. Die Erklärung dieser Tatsache dürfte vielleicht in dem Umstand zu suchen sein, daß die einzelnen Tiere eine mehr oder weniger große Empfänglichkeit für Infektion besitzen. Dabei ist jedenfalls auch die bakterizide Eigenschaft des Blutes nicht außer Acht zu lassen.

Aus der zweiten Versuchsreihe geht hervor, daß man von einer Disposition des Appendix zu hämatogenen Entzündungen kaum sprechen kann, indem bei Injektion von infektiösem Material in die Art. mesent. sup. auch andere Darmabschnitte in ähnlicher Weise affiziert werden, wie der Appendix.

Dieses Ergebnis der Experimente stimmt mit den Erfahrungen, welche man bei der so häufigen spontanen Appendicitis des Menschen zu machen Gelegenheit hat, überein. Hier nimmt man im allgemeinen eine Infektion vom Darmlumen aus an, und zwar wird dabei das exklusive Befallensein des Appendix nicht auf den histologischen $\mathrm{Zu}$ sammenhang der Wand, sondern auf die groben anatomischen Verhältnisse des Appendix zurückgeführt. Was die histologisehen Verhältnisse der Wand anbelangt, so finden wir dieselben auch in anderen Darmabschnitten, ohne daß es hier zu dem Prozesse kommt, den wir beim Appendix zu sehen gewohnt sind. Dagegen sind die groben anatomischen Verhältnisse derart, daß es leicht zur Stagnation des 
Inhaltes und erst im Anschluß an dieselbe zur Infektion vom Darmlumen her kommt.

Auch beim Kaninchen findet man gelegentlich, wie ich a. a. $0 .{ }^{1)}$ gezeigt habe, spontane Appendicitis wie beim Menschen. Im zweiten Fall war der Sac. rot. (s. oben: Textfigur) auch affiziert, bei diesem kann es seiner anatomischen Beschaffenheit nach unter Umständen leicht zur Stagnation des Inhaltes kommen. Dieser Umistand spricht dafür, daß dem Zustandekommen einer Infektion des Appendix beim Kaninchen ähnliche Verhäitnisse zugrunde liegen können, wie beim Menschen. Allerdings scheint die spontane Appendicitis beim Kaninchen selten zu sein, denn ich hatte seit der Drucklegung der angeführten Publikation keine Gelegenheit gehabt, dieselbe zu beobachten, trotz der relativ großen Zahl von sezierten Kaninchen.

Es war denn auch nach den klinisch bei Menschen gemachten Erfahrungen a priori im höchsten Grade wahrscheinlich, daß der Versuch ein negatives Resultat haben würde, beim Tiere den Nachweis zu erbringen, daß dessen Processus vermiformis besonders disponiert ist für das Auftreten von hämatogener Infektion. Dagegen sind die gemachten Versuche insofern von Interesse, als sie bezüglich des Verkaltens des injizierten Materials einigen Aufschluß geben.

Von Bedeutung sind in dieser Beziehung die Versuche 18, 31, 42, 52 und 54. Bei denselben können wir zwei Gruppen unterscheiden. In einer Gruppe, die Versuche 18 und 54 umfassend, fanden sich die injizierten Bakterien sowohl im Herde, als auch im Herzblut vor. Bei der zweiten Gruppe, zu welcher die Versuche 31,42 und 52 gehören, konnten injizierte Bakterien weder im Herzblut noch in dem Herd des betreffenden Darmstïckes nachgewiesen werden. Dagegen fand man bei sämtlichen Versuchen in den Herden der Darmwand konstant Bakterien, die nur aus Darminhalt stammen konnten.

Entsprechend diesem verschiedenen Verhalten der injizierten Bakterien ist auch der Krankheitsverlauf in den beiden Gruppen von Versuchen ein verschiedener. In der ersten Gruppe führte Injektion von Bakterien in die Art. mesent. sup. außer zur Entstehung von Herden in der Darmwand zur Bakteriämie und infolgedessen zu einem raschen Exitus. In der zweiten Gruppe bestanden die Folgen der Injektion von Bakterien nur in dem Auftreten von Entzündungsherden in der Darmwand. Daher war der Krankheitsverlauf ein protrahierter. Dabei gingen die injizierten Bakterien in den betreffenden Herden

1) Cf. M. Mori, Spontane Appendicitis bei einigen Tierspezies. Mitteilungen aus den Grenzgebieten zwischen der Medizin und Chirurgie. 1903. 12. Bd. Heft 5. S. 641 . 
136 VI. MoRI, Eine experimentelle Arbeit über die Ätiologie der Perityphlitis. zugrunde, und an ihre Stelle traten Bakterien, welche aus dem Darminhalte stammten.

Aus diesem Befunde ergibt sich die Frage, ob diesen eingewanderten Bakterien bezüglich der weiteren Entwickelung der Entzündung der Darmhaut eine Bedeutung zukommt oder nicht. Leider müssen wir diese Frage in suspenso lassen, da unsere Versuche in diesen Beziehungen keinen Anhaltspunkt geben, um ihr gegentiber Stellung. zu nehmen.

Folgendes Resumé scheint sich mir aus den gefundenen Resultaten mit aller Deutlichkeit zu ergeben:

1. Perityphlitis kann auch von dem Blutwege aus entstehen.

2. Für die hämatogene Entstehung bietet das Kaninchen keine günstigen Verhältnisse.

3. Obige Experimente lassen die Auffassung gerechtfertigt erscheinen, daß auch beim Menschen die intestinale Form der Perityphlitis gegenüber der hämatogenen die häufigere sei.

Zum Schluß sei es mir gestattet, meinem hochverehrten Lehrer Herrn Prof. Dr. Theodor Kocher für die Anregung zur Bearbeitung dieses Themas meinen aufrichtigen Dank auszusprechen. 